\title{
Genetische Analyse des individuellen Wachstums- und Futteraufnahmeverlaufs von Jungebern während der Eigenleistungsgruppenprüfung
}

\author{
Herrn Professor Dr. Erhard Kallweit zum 65. Geburtstag gewidmet
}

\begin{abstract}
Summary
Title of the paper: Genetic analysis of the course of individual growth and feed intake of group-penned performance tested boars

Objective of the study was to analyse the course of feed intake and growth of 661 boars of three lines $(259,208$. and 194 animals of lines 3,4 , and 8 , respectively) during an age dependent performance test between the $100^{\mathrm{th}}$ and $170^{\text {th }}$ day of age. Individual feed intake of the group-penned animals was recorded by electronic feeding stations during the first, third, fifth, seventh, and ninth week on test. Additionally, each animal was weighted in biweekly intervalls. A second order polynomial and a third order polynomial were individually fitted on feed intake and live weight. From these curves, individual information about daily feed intake, daily gain, and food conversion ratio were derived for five periods of 12 days and for the entire period. Genetic parameters were estimated simultaneously using a multiple trait animal model. Heritabilities of $\mathrm{h}^{2}=.50, .55$, .40 , and .39 for traits of entire test period were found for backfat thickness, daily gain, daily feed intake, and food conversion ratio, respectively. Estimated heritabilities for each test period from one to five were $h^{2}=.50, .56, .54, .50$, and .37 for daily gain, $h^{2}=.18, .43, .46, .45$, and .38 for daily feed intake, and $h^{2}=.34, .42, .46, .44$, and .39 for food conversion. The genetic correlation between daily gain and food conversion in period one $\left(r_{8}=-84\right)$ was significantly different from correlations between these traits in the following periods $\left(r_{g}=-.32\right.$ to $\left.-.5 I\right)$. Genetic correlations between daily gain and daily feed intake in each period were $r_{B}=.56$ to .42 from first to last period. Except of first period $\left(\mathrm{r}_{\mathrm{g}}=-.1 \mathrm{I}\right)$, the genetic associations between food conversion and daily feed intake for periods were similar $\left(r_{8}=.52\right.$ to .56$)$. The genetic associations indicate, that a high feed intake in the beginning of the fattening period is desirable, while afterwards a more reduced feed intake should be achieved to improve efficiency of fattening performance. In order to optimise fattening performance, the use of part test information from growth rate and feed intake is necessary.
\end{abstract}

Key Words: growth curve, feed intake curve, pigs, heritability, genetic correlation

\section{Zusammenfassung}

Ziel der Untersuchung war die Analyse des Verlaufs der Futteraufnahme und des Wachstums von 661 Jungebern (259, 208 und 194 Tiere) der Linien 3,4 und 8 während einer altersbezogenen Eigenleistungsprufung zwischen dem 100. und 170. Lebenstag. Individuelle Futteraufnahmeinformationen der in Gruppen gehaltenen Tiere wurden in der ersten, dritten, funften, siebten und neunten Testwoche mit elektronischen Futterstationen aufgezeichnet. Zusätzlich wurden die Tiergewichte im 14-tägigen Abstand erfasst. Die individuelle Futteraufnahme und Lebendmasseentwicklung wurde mit Polynomen zweiten und dritten Grades beschrieben. Aus diesen Funktionen wurden Informationen uber die Futteraufnahme, Tageszunahme und Futterverwertung fur funf Perioden von 12 Tagen und für die gesamte Periode abgeleitet. Die genetischen Parameter wurden auf Basis eines Mehrmerkmals-Tiermodells multivariat geschătzt. Uber den gesamten Zeitraum wurden Heritabilitaten von $\mathrm{h}^{2}=0,50,0,55$, 0,40 und 0,39 für Rückenspeckdicke, Tageszunahme, Futteraufnahme und Futterverwertung geschätzt. Die Heritabilitäten in den Perioden eins bis funf betrugen $h^{2}=0,50,0,56,0,54,0,50$ und 0,37 für die Tageszunahme, $h^{2}$ $=0,18,0,43,0,46,0,45$ und 0,39 für die Futteraufnahme sowie $h^{2}=0,34,0,42,0,46,0,44$ und 0,38 fur die Futterverwertung. Die genetischen Korrelationen zwischen Tageszunahme und Futterverwertung in der Periode eins. 
$\left(T_{\mathrm{g}}=-0,84\right)$ unterschieden sich signifikant von denen in den folgenden vier Perioden $\left(r_{\mathrm{B}}=-0,32\right.$ bis $\left.-0,51\right)$, Zwischen der Tageszunahme und der Futteraufnahme wurden von der ersten bis zur funften Periode Korrelationen von $r_{\mathrm{g}}=0,56$ bis 0,42 geschatzt. Die Futterverwertung zeigte bis auf die erste Periode $\left(r_{\mathrm{B}}=-0,11\right)$ fast identische Beziehungen zur entsprechenden Futteraufnahme $\left(r_{\mathrm{g}}=0,52\right.$ bis 0,56$)$. Die genetischen Beziehungen zeigen an, dass am Anfang der Mast eine hohe Futteraufnahme und mit Fortdauer der Mast eine Limitierung der Futteraufnahme anzustreben ist, um die Effizienz der Mastleistung zu erhøhen. Um die Mastleistung zu optimieren, ist daher die Verwendung von Abschnittsinformationen des Wachstums und der Futteraufnahme notwendig.

Schlusselworter: Wachstumskurve, Futteraufnahmekurve, Schweine, Heritabilitat, genetische Korrelation

\section{Einleitung}

Zur Optimierung der Mastleistung von Schweinen ist neben der Kenntnis des Verlaufs der Futteraufnahme die Kenntnis über die Entwicklung des Wachstums von entscheidender Bedeutung, um die Effizienz des Einsatzes von Futter beurteilen zu können und dies in den Selektionsentscheidungen zu berücksichtigen (KNAP und VAN DER STEEN, 1994). DE HAER (1992) verwendete als Effizienzparameter die Restfutteraufnahme, die zu Mastende positiv war, während am Anfang der Mastperiode der Bedarf zur Ausnutzung des Wachstumspotentials durch die freiwillige Futteraufnahme nicht gedeckt werden konnte. KRIETER (1986) empfiehlt die Berücksichtigung der Muskelfleischwachstumsrate, um einer Reduktion der Futteraufnahme durch eine Selektion auf Fleischreichtum entgegenzuwirken.

Während die Futteraufnahme im Verlauf der Mast hinreichend gut mit linearen Regressionen ersten oder zweiten Grades auf das Alter bzw. Gewicht erfassbar ist (EISSEN, 2000; SCHMIDT et al,, 1991), wurden zur Beschreibung des Wachstumsverlaufs lineare Funktionen höherer Ordnung verwendet (SCHMIDT, 1988; ANDERSEN und PEDERSEN, 1996).

Die individuelle Erfassung der Futteraufnahme in Gruppenhaltung ist seit der Einfuhrung von elektronischen Futterstationen möglich und ist hinsichtlich der Verwendungsmöglichkeiten in den letzten Jahren mehrfach untersucht worden (DE HAER, 1992; VON FELDE et al., 1996; LABROUE et al. 1997; HALL et al. 1999; EISSEN, 2000). Dabei wurde die Gewichtsentwicklung jedoch nur uber den gesamten Prüfzeitraum ausgewertet. Angaben uber genetische Parameter von Tageszunahmen und Futteraufnahme in Teilabschnitten sind bei KRIETER (1986) und SCHMIDT (1988) zu finden. Jedoch wurde in diesen Arbeiten die individuelle Futteraufnahme aus der mittleren Futteraufnahme der Gruppe (2 Tiere/Gruppe) und der individuellen Zunahmeleistung geschätzt. Ausserdem war zu der Zeit keine Schätzung der genetischen Parameter auf der Basis eines Tíermodells möglich.

Ziel der Untersuchung war die simultane Analyse der individuellen Futteraufnahmeund Wachstumskurve von Jungebern während einer Eigenleistungsprüfung. Des Weiteren wurden die genetischen Beziehungen zwischen Abschnittsleistungen in den Merkmalen Tageszunahme, Futteraufnahme und Futterverwertung sowie die Beziehungen zu den Mastleistungsmerkmalen des gesamten Zeitraums analysiert.

\section{Material und Methoden}

Für die vorliegende Untersuchung lagen Mastleistungsergebnisse und Informationen über die Gewichtsentwicklung und Futteraufnahme von 661 (259, 208 und 194) Jungebern der Mutterlinien 3, 4 und 8 vor. Diese Tiere stammten von 122 Vätem und 407 
Müttern ab, Die Daten wurden zwischen August 1997 und April 1998 in der zentralen Eigenleistungsprufstation eines norddeutschen Zuchtunternehmens erhoben.

Die Probanden wurden zur Eingewöhnung zehn Tage vor Testbeginn aus einer vorgelagerten Aufzuchtstation, in der die Ferkel seit dem 21. Lebenstag aus mehreren Nucleusbetrieben gemeinsam aufgezogen wurden (ISOWEAN), in die Prufanstalt umgestallt. Während der zehn Wochen dauernden, altersabhängigen Eigenleistungsprüfung (100. bis 170. Lebenstag) wurden die Probanden in Gruppen von 12 Tieren der gleichen Linie auf Stroh gehalten. Die Fütterung erfolgte ad libitum mit einem pelletierten Trockenfutter (12,6 MJ umsetzbare Energie, $18 \%$ Rohprotein, $1 \%$ Lysin je kg Futtermittel). Die individuelle Futteraufnahme wurde mit elektronischen Futterstationen des Typs Acema48 mit Einzeltieridentifikation in der ersten, dritten, fünften, siebten und neunten Testwoche erfasst. In den verbleibenden Testwochen fand bei 410 Tieren keine Aufzeichnung des individuellen Futterverzehrs statt, während bei 251 Probanden Futteraufnahmeinformationen auch in diesen Testwochen vorlagen. Diese Informationen wurden jedoch nicht in der Auswertung berlicksichtigt, um eine Gleichbehandlung aller Tiere zu gewährleisten. Der Einfluss des Fütterungsmodus wurde in einer anderen Untersuchung analysiert und wird nachfolgend im statistischen Modell als fixer Effekt berücksichtigt.

Am Ende der Leistungsprllung wurden die Probanden gewogen sowie drei Speckmaße an Kotelett und Lende eindimensional mittels Ultraschall gemessen (RENCOLeanmeater). Während der Leistungsprufung wurden alle Probanden im 14-tägigen Abstand gewogen, so dass zusätzlich zum Anfangs- und Endgewicht vier Gewichte während der Prüfung von jedem Jungeber vorlagen. An die Futteraufnahmeinformationen wurden entsprechend vorheriger Untersuchungen (SCHULZE et al., 2000) nachfolgende Anforderungen gestellt. Aufgrund festgestellter Adaptionseffekte an die elektronischen Futterstationen wurden die Futteraufnahmeinformationen der ersten zwei Testtage einer jeden neuen Testperiode nicht berllcksichtigt. Ausserdem mussten in jeder der funf Testwochen 1, 3, 5, 7 und 9 Aufzeichnungen vorhanden sein. Weiterhin musste die mittlere gemessene Futteraufnahme einer jeden Testwoche über dem berechneten Erhaltungsbedarf liegen. Der individuelle Bedarf an umsetzbarer Energie in Megajoule (MJME) wurde aus dem täglichen Gewichtszuwachs zwischen zwei Wiegungen entsprechend der Formel $0,719^{*}$ Lebensmasse $^{0,63}$ (ARC, 1981) abgeleitet sowie auf das Futtermittel umgerechnet und zu Wochenmittelwerten für die erwartete Futteraufnahme in $\mathrm{g}$ verdichtet. Die Mittelwerte und Standardabweichungen fur Mastleistungsmerkmale und fur jede Wiegung sind in Tabelle 1 dargestellt.

Die hohe Priftagszunahme von $971 \mathrm{~g}$ bei 10,2 mm Ruckenspeck erreichten die Probanden mit einer Futteraufnahme von annähernd 2,5 kg. Die Rückenspeckdicke und die Futteraufnahme weisen mit 17 und $14 \%$ die hochsten Variationskoeffizienten auf. Bei den einzelnen Gewichtsmerkmalen beträgt die Variation 9 bis $10 \%$ mit maximalen Variationskoeffizienten bei der zweiten und dritten Wiegung. Der höchste Lebendmassezuwachs von $14,6 \mathrm{~kg}$ wird zwischen der vierten und fünten Wiegung erreicht. Sowohl die aus den Gewichtsinformationen abgeleiteten Tageszunahmen für die Prüfabschnitte von 14 Tagen als auch die mittlere Futteraufnahme in den einzelnen Testwochen sind in Tabelle 2 dargestellt. Die Tageszunahmen steigen von $884 \mathrm{~g} \mathrm{im}$ ersten Abschnitt mit abnehmenden Zuwachsraten bis zu einem Maximum von $1043 \mathrm{~g}$ 
im vierten Abschnitt an. Im letzten Abschnitt liegt die mittlere Zunahme bei $975 \mathrm{~g}$, jedoch ist die Standardabweichung gegenüber der in den vorherigen Abschnitten erhöht. Die Futteraufnahme steigt während der gesamten Prüfung von $1763 \mathrm{~g}$ auf über $3049 \mathrm{~g}$ im letzten Abschnitt an.

Tabelle I

Mittelwerte $(\bar{x})$, Standardabweichungen $(s \bar{x})$ und Variationskoeffizienten (VK) fur Mastleistungsmerkmale und Gewichtsentwicklungen wăhrend der Leistungsprllung (Means $(\bar{x})$, standard deviations $(s \bar{x})$, and coefficient of variation (VK) of fattening performance traits and the developement of weight during performance test)

\begin{tabular}{llllc}
\hline Merkmal & & $\bar{x}$ & $s \bar{x}$ & VK \% \\
\hline Tageszunahme (TZ) & $(\mathrm{g})$ & 971 & 107 & 11 \\
Ruckenspeckdicke (RS) & $(\mathrm{mm})$ & 10,2 & 1,76 & 17 \\
Futteraufnahme (FUA) & $(\mathrm{g})$ & 2496 & 347 & 14 \\
Futterverwertung (FVW) & $(\mathrm{g} / \mathrm{kg})$ & 2579 & 315 & 12 \\
Anfangsgewicht & $(\mathrm{kg})$ & 45,1 & 4,2 & 9 \\
2. Wiegung & $(\mathrm{kg})$ & 57,5 & 5,9 & 10 \\
3. Wiegung & $(\mathrm{kg})$ & 70,9 & 6,9 & 10 \\
4. Wiegung & $(\mathrm{kg})$ & 84,9 & 7,8 & 9 \\
5. Wiegung & $(\mathrm{kg})$ & 99,5 & 8,6 & 9 \\
Endgewicht & $(\mathrm{kg})$ & 112,0 & 9,3 & 8 \\
\hline
\end{tabular}

Tabelle 2

Mittelwerte $(\bar{x})$ und Standardabweichungen $(s \bar{x})$ fur Futteraufnahme und Tageszunahmen in den Prufabschnitten zu je 2 Wochen (Means $(\bar{x})$ and standard deviations $(s \bar{x})$ for daily gain and daily feed intake in biweekly test periods)

\begin{tabular}{|c|c|c|c|c|c|}
\hline \multirow[t]{3}{*}{ Merkmal } & \multicolumn{5}{|c|}{ Prufabschnitt } \\
\hline & Abschnitt 1 & Abschnitt 2 & Abschnitt 3 & Abschnitt 4 & Abschnitt 5 \\
\hline & $\bar{x} \pm s \bar{x}$ & $\bar{x} \pm s \bar{x}$ & $\bar{x} \pm \mathrm{s} \bar{x}$ & $\bar{x} \pm s \bar{x}$ & $\bar{x} \pm s \bar{x}$ \\
\hline ØAlter & $105 \pm 2,9$ & $119 \pm 2,9$ & $133 \pm 2,9$ & $147 \pm 2,9$ & $161 \pm 2,9$ \\
\hline$\varnothing$ Gewicht ${ }^{1)} \mathrm{kg}$ & $51,1 \pm 4,9$ & $63,9 \pm 6,3$ & $77,5 \pm 7,2$ & $91,7 \pm 8,1$ & $104,8 \pm 8,6$ \\
\hline Tageszunahme ${ }^{11} \mathrm{~g}$ & $884 \pm 227$ & $954 \pm 232$ & $1000 \pm 241$ & $1043 \pm 211$ & $975 \pm 273$ \\
\hline Futteraufnahme ${ }^{2)_{\mathrm{g}}}$ & $1763 \pm 507$ & $2215 \pm 540$ & $2464 \pm 581$ & $2843 \pm 571$ & $3049 \pm 647$ \\
\hline
\end{tabular}

\section{Funktionen zur Beschreibung des Zunahme- und Futteraufnahmeverlaufs}

Der Verlauf der Futteraufnahme wurde auf Basis einer vorherigen Untersuchung (SCHULZE et al,, 2000) tierindividuell als Funktion des Alters mit einer linear-quadratischen Regression geschätzt. Die durchschnittliche geschätzte Futteraufnahme wurde aus der Stammfunktion für fünf Perioden von jeweils 12 Tagen und uber die gesamte Prüfperiode tierindividuell abgeleitet. Diese Perioden wurden gewählt, um den vollständigen Zeitraum mit Futteraufnahmeaufzeichnungen abzudecken.

Zur Beschreibung der Lebendmasseentwicklung als Funktion des Alters erwies sich ein Polynom dritten Grades als geeignet. Analog zur Futteraufnahme wurden identische Perioden gewählt, für die aus dem Verlauf der Steigung der Funktion die durchschnittlichen Tageszunahmen tierindividuell für die fünf Perioden und die gesamte Prüfperiode bestimmt wurden.

Die Mittelwerte für die tierindividuellen Funktionsparameter sind in Tabelle 3 dargestellt. Für die Futteraufnahme konnte ein mittleres Bestimmtheitsmaß von $\mathrm{R}^{2}=36,5 \%$ erzielt werden. Für die Gewichtsentwicklung zeigte das kubische Polynom die beste Anpassung $\left(\mathrm{R}^{2}=99,8 \%\right)$. 
Tabelle 3

Mittelwerte $(\bar{x})$ und Standardabweichungen $(s \bar{x})$ der Funktionsparameter aus individuelter polynomialer Anpassung der Lebendmasse und der Futteraufnalume als Funktion des Alters (Pooled means $(\bar{x})$ and standard deviations $(s \bar{x})$ of parameters after individually fitting functions for feed intake and live weight in relation to age)

\begin{tabular}{|c|c|c|c|}
\hline $\begin{array}{l}\text { Merkmal } \\
\text { Funktion } \\
\text { Parameter } \\
\end{array}$ & & $\begin{array}{c}\text { Futteraufnahme } \\
\text { Polynom } 2 \text {. Grades } \\
\bar{x} \pm s \bar{x} \\
\end{array}$ & $\begin{array}{c}\text { Lebendmasse } \\
\text { Polynom } 3 \text {. Grades } \\
\bar{x} \pm s \bar{x} \\
\end{array}$ \\
\hline Bestimmtheitsmaß $\left(\mathrm{R}^{2}\right)$ & $\%$ & $36,5 \pm 20$ & $99,8 \pm 0,3$ \\
\hline Restvarianz (RSD) & $\mathrm{g}$ & $759,6 \pm 193,3$ & $1520 \pm 880$ \\
\hline Anfangswert ${ }^{1}$ & $\mathrm{~g}$ & $1701,6 \pm 563,7$ & $46800 \pm 4500$ \\
\hline Linearer Term $\quad\left(b_{1}\right)$ & $\mathrm{g}$ & $58,45 \pm 191,6$ & $-961 \pm 5840$ \\
\hline Quadratischer Term $\left(b_{2}\right)$ & $\mathrm{g}$ & $-0,135 \pm 0,74$ & $13,9 \pm 0.045$ \\
\hline Kubischer Term $\quad\left(b_{3}\right)$ & $\mathrm{g}$ & - & $-0,0324 \pm 0,11$ \\
\hline
\end{tabular}

Die Entwicklungen der mittleren geschätzten Futteraufnahme, der mittleren geschätzten Tageszunahme und der aus diesen beiden Informationen abgeleiteten geschätzten Futterverwertung zwischen dem 100. und 160. Lebenstag sind in Abbildung 1 dargestellt.

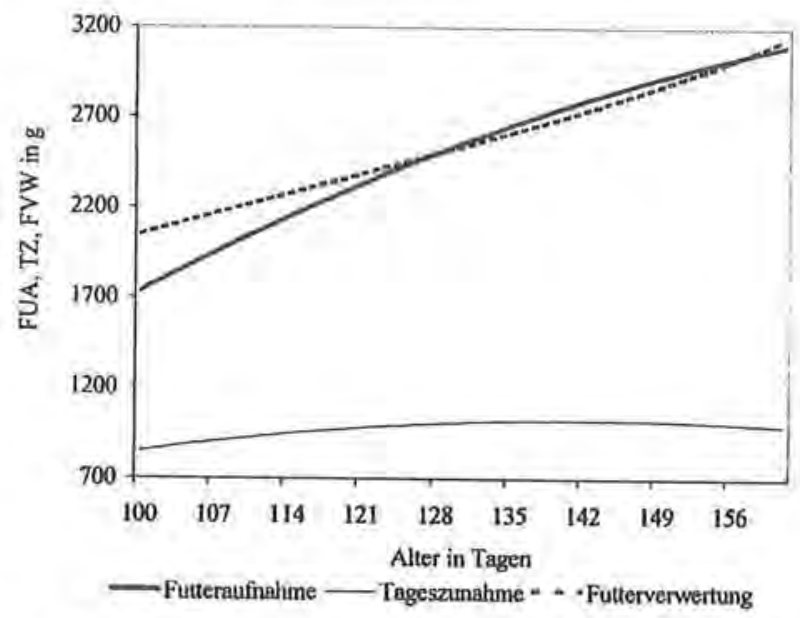

Abb. 1: Verlauf der geschátzten Futteraufnahme (FUA) und Tageszunahme (TZ) sowie der daraus abgeleiteten Futterverwertung (FVW) als Funktionen des Alters der Tiere wăhrend der Leistungspruffung (Developement of estimated daily feed intake (FUA), daily gain (TZ) as well as of food conversion ratio (FVW) as functions of age during performance test)

Die Tageszunahme zeigt einen kurvenlinearen Verlauf mit maximalen Werten zwischen dem 135. und 149. Lebenstag der Tiere. Die Futteraufnahme steigt mit abnehmenden Zuwachsraten an, wăhrend die Futterverwertung uber den gesamten Zeitraum einen linearen Zusammenhang mit der Altersentwicklung andeutet.

Zur Verdeutlichung dieser Verläufe sind in Tabelle 4 die Mittelwerte und Standardabweichungen der aus den Funktionen abgeleiteten Tageszunahmen und Futteraufnahmen für die einzelnen Perioden dargestellt. Die geschätzte Tageszunahme steigt von $883 \mathrm{~g}$ in Periode 1 auf maximale Zunahmen von $1021 \mathrm{~g}$ in der vierten Periode an. 
Tabelle 4

Mittelwerte $(\bar{x})$ und Standardabweichungen $(s \bar{x})$ fur die geschätzte durchschnittliche Futteraufnahme, geschätzte durchschnittliche Zunahme in Perioden von je 12 Tagen und den abgeleiteten Merkmalen zur Beschreibung der Effizienz des Wachstums (Means $(\bar{x})$ and standard deviations $(s \bar{x})$ of average estimated feed intake, average estimated daily gain for periods of 12 days each and other derived traits to describe efficiency of growth)

\begin{tabular}{lcccccc} 
Merkmal $^{15}$ & \multicolumn{5}{c}{ Testperiode } \\
& 1 & 2 & 3 & 4 & 5 & $1-5$ \\
& $\bar{x} \pm \mathrm{s} \bar{x}$ & $\bar{x} \pm \mathrm{s} \bar{x}$ & $\bar{x} \pm \mathrm{s} \bar{x}$ & $\bar{x} \pm \mathrm{s} \bar{x}$ & $\bar{x} \pm \mathrm{s} \bar{x}$ & $\bar{x} \pm \mathrm{s} \bar{x}$ \\
\hline TZ* $^{*}$ & $883 \pm 207$ & $957 \pm 149$ & $1003 \pm 169$ & $1021 \pm 153$ & $1011 \pm 163$ & $975 \pm 112$ \\
FUA $^{*}$ & $1883 \pm 435$ & $2220 \pm 426$ & $2518 \pm 471$ & $2778 \pm 448$ & $2998 \pm 529$ & $2478 \pm 359$ \\
FVW $^{*}$ & $2232 \pm 725$ & $2340 \pm 407$ & $2546 \pm 478$ & $2752 \pm 445$ & $3017 \pm 608$ & $2552 \pm 311$ \\
FUA $_{\text {Erthaliung }}$ & $688 \pm 41$ & $778 \pm 46$ & $865 \pm 51$ & $950 \pm 53$ & $1032 \pm 54$ & $862 \pm 48$ \\
FUA $_{\text {Leistung }}$ & $1195 \pm 423$ & $1442 \pm 406$ & $1653 \pm 448$ & $1828 \pm 422$ & $1966 \pm 509$ & $1617 \pm 335$ \\
\hline
\end{tabular}

") Abkurzungen und Einheiten siehe Tabelle 1

* Merkmal abgeleitet aus der entsprechenden polynomialen Funktion

In der letzten Periode ist ein leichter Abfall in der Wachstumsrate zu beobachten, der jedoch schwächer ausfallt als bei den direkt aus den Wiegungen bestimmten $\mathrm{Ab}$ schnittszunahmen in Tabelle 2. Die Futteraufnahme steigt kontinuierlich an von 1883 $\mathrm{g}$ auf $2998 \mathrm{~g}$ von der ersten bis zur letzten Periode an. Im Vergleich zu den entsprechenden Abschnitten für Tageszunahme und Futteraufnahme in Tabelle 2 sind die Standardabweichungen vermindert. Die Futterverwertung zeigt die höchste Effizienz in der ersten Periode mit einem mittleren Wert von $2232 \mathrm{~g}$. In den folgenden Perioden steigt die Futterverwertung mit Zuwachsraten von $108 \mathrm{~g}, 206 \mathrm{~g}, 206 \mathrm{~g}$ und $265 \mathrm{~g}$ an. Auffallig sind die höheren Standardabweichungen in der ersten und funften Periode gegenüber den mittleren Abschnitten. Für den gesamten Zeitraum zeigen die aus den Funktionen abgeleiteten Mittelwerte furr Tageszunahme, Futteraufnahme und Futterverwertung mit $975 \mathrm{~g}, 2478 \mathrm{~g}$ und $2552 \mathrm{~g}$ eine gute Öbereinstimmung mit den Mittelwerten der Messwerte in Tabelle 1.

\section{Statistische Analysen}

Die Analyse der fixen Umweltfaktoren wurde mit der SAS-Statistikprozedur GLM durchgefuhrt. Das dabei verwendete Modell beinhaltete die fixen Einflussfaktoren der Linie, des Geburtsbetriebs, der Saison sowie des Fütterungssystems. Das individuelle Startgewicht zu Testbeginn wurde als lineare Regression auf das durchschnittliche Anfangsgewicht berucksichtigt. Einziger zufäliger Effekt war neben dem genetischen Einfluss des Tieres der Restfehler. Das genetisch-statistische Modell besteht aus:

$$
\mathbf{Y}_{\mathrm{j} \mathrm{jlm}}=\mu_{\mathrm{m}}+\mathbf{L}_{\mathrm{im}}+\mathrm{S}_{\mathrm{jm}}+\mathbf{B}_{\mathrm{km}}+\mathbf{F}_{\mathrm{lm}}+\mathbf{b}_{\mathrm{m}}{ }^{*}\left(\mathbf{X}_{\mathrm{ijklm}}-\overline{\mathbf{x}}\right)+\mathbf{a}_{\mathrm{ijk \textrm {m }}}+\mathbf{e}_{\mathrm{ijklm}}
$$

dabei bedeuten

$\mathrm{Y}_{\mathrm{ijklm}}=$ individuelle Beobachtungswerte im m-ten Merkmal

$\mu_{\mathrm{m}} \quad=$ Populationsmittelwert im m-ten Merkmal

$\mathrm{L}_{\mathrm{im}}=$ fixer Effekt der i-ten Linie

$\mathrm{S}_{\mathrm{jm}} \quad=$ fixer Effekt der $\mathrm{j}$-ten Saison

$B_{k \mathrm{~m}}=$ fixer Effekt des k-ten Geburtsbetriebes

$\mathrm{F}_{\mathrm{Im}}$ = fixer Effekt des 1-ten Fütterungsmodus

$$
\begin{aligned}
& (\mathrm{i}=1,2,3) \\
& \mathrm{j}=1,2,3) \\
& (\mathrm{k}=1,2,3) \\
& (1=1,2)
\end{aligned}
$$


Arch. Tierz, 44 (2001) 2

$\mathrm{b}_{\mathrm{m}} \quad=$ lineare Regression des individuellen Startgewichts $\left(\mathrm{x}_{\mathrm{ijk} \mathrm{km}}\right)$ auf das mittlere Startgewicht $(\bar{x})$

$\mathrm{a}_{\mathrm{ijklm}}=$ zufälliger genetischer Tiereffekt

$\mathrm{e}_{\mathrm{ijkllm}}=$ Restfehler

Die Varianzkomponentenschätzung erfolgte multivariat mit dem REML-Programm MTDFS (MISZTAL, 1993) unter Berïcksichtigung der aufgeführten Effekte auf Basis eines Mehrmerkmals-Tiermodells. Die Standardfehler der genetischen Korrelationen wurden approximativ nach der Methode von ROBERTSON (1959) berechnet.

\section{Ergebnisse}

\section{Einfluss von Zunahme- und Futteraufnahmeverlauf auf die Mastleistung}

Auf Basis der um die im Modell beschriebenen Faktoren korrigierten Merkmale wurde eine Einteilung des Datensatzes anhand der Mediane für tägliche Zunahme $(975 \mathrm{~g})$ und Rückenspeckdicke $(10,1 \mathrm{~mm})$ vorgenommen, um sowohl den Verlauf der geschätzten Futteraufnahme als auch den von Tageszunahme und Futterverwertung bei unterschiedlichen Leistungsniveaus zu charakterisieren. Daraus ergaben sich vier Gruppen, für die in den Abbildungen 2 bis 4 die Verläufe als Funktionen des Alters dargestellt sind.

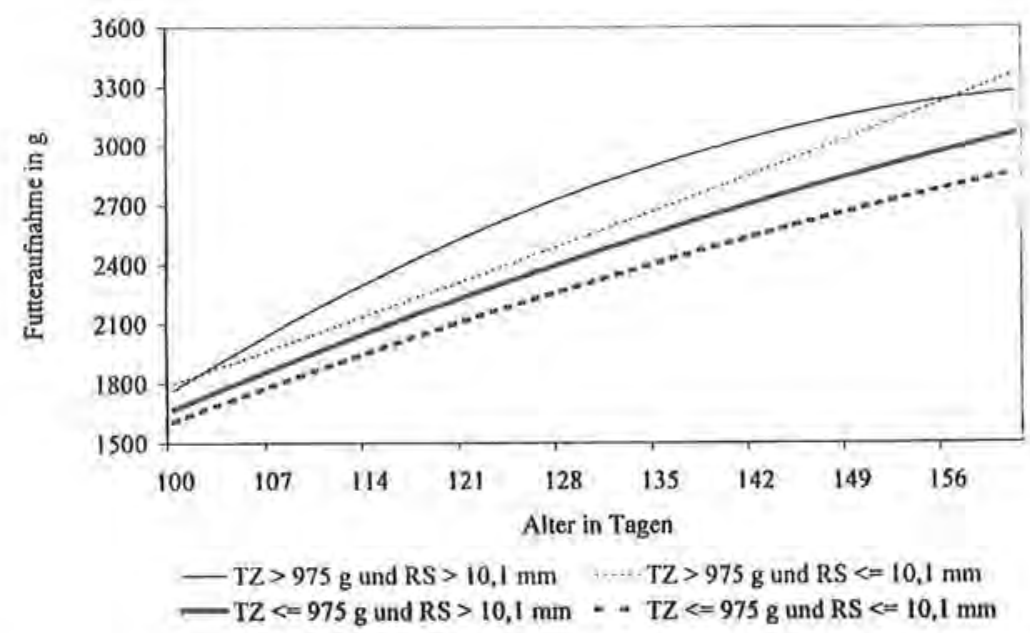

Abb. 2: Futteraufnahme als Funktion des Alters bei unterschiedlichen Leistungsniveaus fur Tageszunahme (TZ) und Rückenspeckdicke (RS) (Feed intake as a function of age for different levels of performances in daily gain (TZ) and backfat thickness (RS))

Abbildung 2 verdeutlicht, dass ein hohes Zunahmeniveau (dünne Linien), unabhängig von der Rúckenspeckdicke, die bei Testende gemessen wird, nur mit einer hohen Futteraufnahme zu Beginn der Leistungsprüfung zu erreichen ist. Die Futteraufnahme der langsam wachsenden Tiere (dicke Linien) liegt am 100, Lebenstag um ca. $200 \mathrm{~g}$ niedriger als die Futteraufnahme der Tiere mit hoher Tageszunahme. Um den 114, Lebens- 
tag sind unabhängig von der Tageszunahme Unterschiede in der Futteraufnahme zwischen mageren (gestrichelte Linien) und speckreichen Tieren (durchgezogene Linien) zu verzeichnen. Auf hohem Zunahmeniveau liegt zwischen den Tieren mit geringer und hoher Speckdicke eine maximale Differenz in der geschätzten Futteraufnahme um den 128. Lebenstag vor, während die Differenz in der geschätzten Futteraufnahme bei den langsam wachsenden Tieren über den gesamten Zeitraum ansteigt. Der Verlauf der Futteraufnahme von Tieren mit geringer Rückenspeckdicke ist annähernd linear, während bei den Tieren mit erhöhter Rückenspeckdicke eine stärkere Krümmung der Futteraufnahmekurve zu beobachten ist.

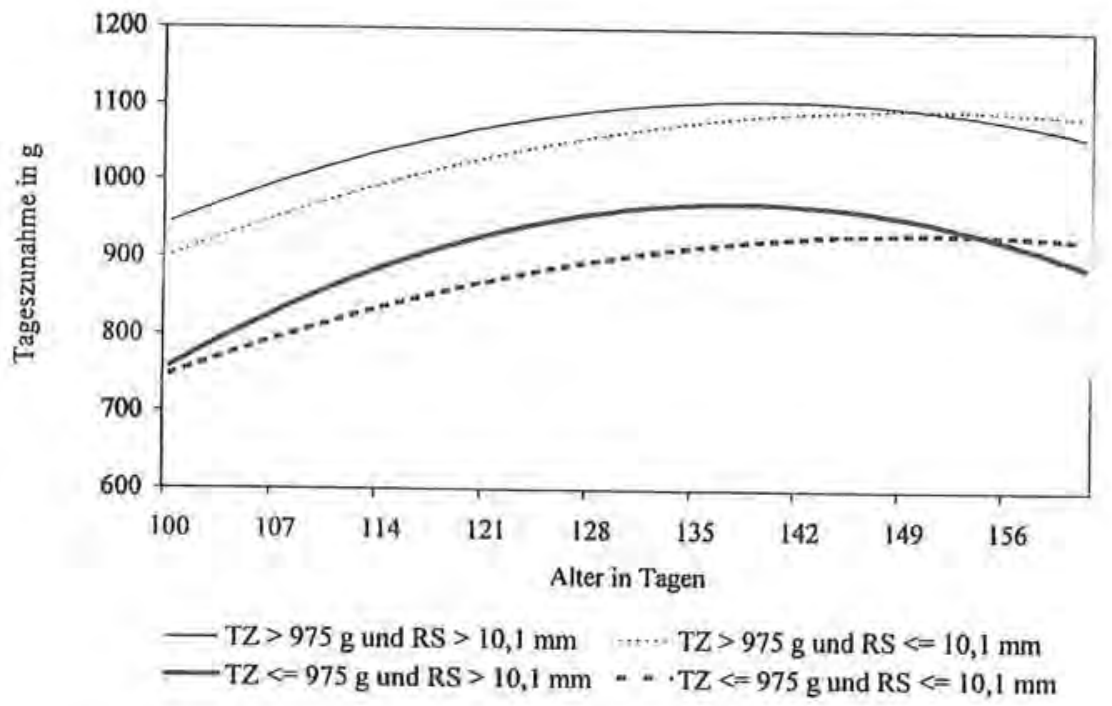

Abb, 3: Tageszunahmen als Funktion des Alters bei unterschiedlichen Leistungsniveaus fur Tageszunahme (TZ) und Rückenspeckdicke (RS) (Daily gain as a function of age for different levels of performances in daily gain (TZ) and backfat thickness (RS))

Der geschätzte Verlauf der Tageszunahme zwischen den vier Leistungsgruppen in Abbildung 3 zeigt eine Parallelverschiebung zwischen langsam (dicke Linien) und schnell wachsenden Tieren (dünne Linien). Die speckreichen Tiere (durchgezogene Linien) weisen eine deutlich stärkere Krümmung in der Zunahmekurve auf als die mageren Tiere (gestrichelte Linien). Die Tiere mit größerer Rückenspeckdicke erreichen ihre maximale Zunahme zwischen den 135. und 142. Lebenstag etwa 14 Tage früher als die mageren Tiere, die ihre maximale Zunahme erst zwischen dem 149. und 156. Lebenstag erreichen. Der Verlauf der Futterverwertung in den einzelnen Leistungsgruppen ist in Abbildung 4 dargestellt.

Mit Ausnahme der ersten Woche wird die niedrigste Futterverwertung über den gesamten Bereich der Leistungsprüfung von den mageren, schnell wachsenden Tieren erzielt. Während die Tiere mit niedrigem Speckmaß eine mehr lineare Entwicklung der Futterverwertung aufweisen, verläuft die Futterverwertung bei den speckreichen Tie- 
ren unterschiedlich. Langsam wachsende, fettreichere Tiere zeigen insbesondere ab dem 142. Lebenstag einen exponentiellen Anstieg der Futterverwertung, während die Tiere mit hohen Zunahmen und erhöhter Rückenspeckdicke (dünne, durchgezogene Linie) zwischen dem 100. und 114. Lebenstag eine sehr gute Futtereffizienz haben. Mit Ausnahme der schnell wachsenden mageren Tiere treten im mittleren Prüfabschnitt um den 130. Lebenstag keine Unterschiede in der Futterverwertung zwischen den Leistungsgruppen auf.

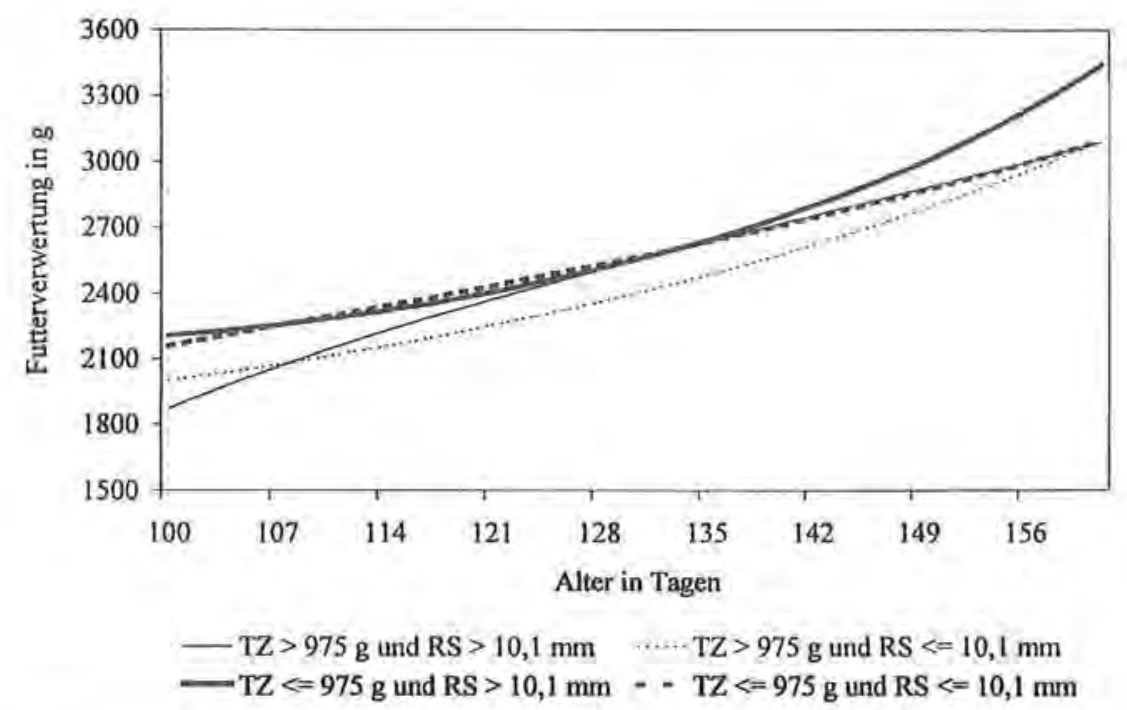

Abb. 4: Entwicklung der Futterverwertung bei unterschiedlichen Leistungsniveaus fur Tageszunahme (TZ) und Ruckenspeckdicke (RS) (Developement of food conversion ratio for different levels of performances in daily gain (TZ) and backfat thickness (RS))

\section{Heritabilitäten fïr die geschätzten Mastleistungsmerkmale}

Die Heritabilitäten für die aus den Funktionen abgeleiteten Mastleistungsmerkmale über die gesamte Untersuchungsperiode und für die einzelnen Perioden sind in Tabelle 5 dargestellt.

Über den gesamten Zeitraum wurden hohe Heritabilitäten von $\mathrm{h}^{2}=0,39,0,40,0,50$ und 0,55 für die Merkmale Futterverwertung, Futteraufnahme, Rückenspeckdicke und Tageszunahme geschätzt. Bei der Betrachtung der Schätzwerte in den einzelnen Perioden zeigt sich für die Futteraufnahme in der ersten Periode ein niedrige Heritabilität $\left(h^{2}=0,18\right)$. Die Erblichkeit der Futteraufnahme steigt bis zur dritten Periode auf $h^{2}=0,46$ an und sinkt in der letzten Prüfperiode geringfugig $a b\left(h^{2}=\right.$ 0,39). Bei den Tageszunahmen sind die geschätzten Heritabilitäten in den ersten vier Perioden hoch $\left(\mathrm{h}^{2}=0,50\right.$ bis 0,56$)$ und sinken in der letzten Periode auf $h^{2}=0,37 \mathrm{ab}$. Für die Futterverwertung wurden hohe Erblichkeiten in den mittleren drei Perioden geschätzt $\left(h^{2}=0,42\right.$ bis 0,46$)$. Die Schätzwerte weisen zu beiden Enden des Beobachtungszeitraums niedrigere Werte auf $\left(h^{2}=0,34\right.$ und 0,38$)$. 
Tabelle 5

Heritabilitaten $\left(h^{2}\right)$ und Standardfehler $\left(s_{h}\right)$ fur die Futteraufnahme, Tageszunahme, Futterverwertung in den einzelnen Perioden und uber alle Perioden sowie für die Rückenspeckdicke (Heritabilities $\left(h^{2}\right)$ and standard errors $\left(\mathrm{s}_{\mathrm{n}}\right)$ for daily feed intake, daily gain, and food conversion for each period and for the entire test period as well as for backfat thickness)

\begin{tabular}{|c|c|c|c|c|}
\hline \multicolumn{2}{|l|}{ Merkmal } & \multirow{2}{*}{$\frac{\sigma_{a}}{174}$} & \multirow{2}{*}{$\frac{\sigma_{e}}{375}$} & \multirow{2}{*}{$\frac{h^{2} \pm s_{b^{2}}}{0,18 \pm 0,09}$} \\
\hline Futteraufnahme & Periode 1 & & & \\
\hline & Periode 2 & 259 & 296 & $0,43 \pm 0,06$ \\
\hline & Periode 3 & 299 & 326 & $0,46 \pm 0,06$ \\
\hline & Periode 4 & 283 & 314 & $0,45 \pm 0,06$ \\
\hline & Periode 5 & 318 & 395 & $0,39 \pm 0,06$ \\
\hline \multicolumn{2}{|c|}{ Gesamtabschnitt } & 210 & 258 & $0,40 \pm 0,06$ \\
\hline \multirow[t]{6}{*}{ Tageszunahme* } & Periode 1 & 143 & 143 & $0,50 \pm 0,05$ \\
\hline & Periode 2 & 106 & 94 & $0,56 \pm 0,04$ \\
\hline & Periode 3 & 114 & 107 & $0,54 \pm 0,05$ \\
\hline & Periode 4 & 101 & 101 & $0,50 \pm 0,05$ \\
\hline & Periode 5 & 98 & 129 & $0,37 \pm 0,07$ \\
\hline & ntabschnitt & 80 & 73 & $0,55 \pm 0,05$ \\
\hline \multirow[t]{5}{*}{ Futterverwertung ${ }^{\circ}$} & Periode 1 & 424 & 585 & $0,34 \pm 0,07$ \\
\hline & Periode 2 & 249 & 292 & $0,42 \pm 0,06$ \\
\hline & Periode 3 & 302 & 330 & $0,46 \pm 0,06$ \\
\hline & Periode 4 & 281 & 315 & $0,44 \pm 0,06$ \\
\hline & Periode 5 & 360 & 465 & $0,38 \pm 0,06$ \\
\hline \multicolumn{2}{|c|}{ Gesamtabschnitt } & 184 & 228 & $0,39 \pm 0,06$ \\
\hline Rückenspeckdicke & & 1,05 & 1,05 & $0,50 \pm 0,05$ \\
\hline
\end{tabular}

- Merkmal abgeicitet aus der entsprechenden polynomialen Funktion

\section{Genetische Parameter zwischen Mastleistungsmerkmalen über den gesamten Zeitraum}

Die genetischen Korrelationen zwischen den Mastleistungsmerkmalen, sowohl die der beobachteten als auch die der aus den Funktionen ableiteten Werte, sind in Tabelle 6 dargestellt. Die abgeleiteten Werte sind mit einem Stern markiert.

Tabelle 6

Genetische Korrelationen $\left(\mathrm{r}_{\mathrm{B}}\right)$ zwischen beobachteten und geschatzten Mastleistungsmerkmalen fur den gesamten Prufzeitraum (Genetic correlations $\left(r_{g}\right)$ between observed and estimated performance traits of the entire test period)

\begin{tabular}{|c|c|c|c|c|c|c|}
\hline Merkmal') & $\begin{array}{c}\text { RS } \\
r_{8} \pm s \mathrm{sg}\end{array}$ & $\begin{array}{c}\text { FUA } \\
r_{\mathrm{g}} \pm \mathrm{s}_{\mathrm{tg}}\end{array}$ & $\begin{array}{l}\text { FVW } \\
r_{3} \pm s_{t 8}\end{array}$ & $\begin{array}{c}\text { FUA }^{*} \\
r_{g} \pm S_{t g}\end{array}$ & $\begin{array}{c}\mathrm{TZ}^{*} \\
\mathrm{r}_{\mathrm{g}} \pm \mathrm{S}_{\mathrm{Ig}}\end{array}$ & $\begin{array}{l}\mathrm{FVW}^{*} \\
\mathrm{r}_{\mathrm{g}} \pm \mathrm{s}_{\mathrm{gg}}\end{array}$ \\
\hline $\mathrm{TZ}$ & $0,39 \pm 0,05$ & $0,74 \pm 0,04$ & $-0,39 \pm 0,09$ & $0,68 \pm 0,04$ & $0,96 \pm 0,00$ & $-0,29 \pm 0,08$ \\
\hline RS & - & $0,51 \pm 0,06$ & $0,16 \pm 0,11$ & $0,47 \pm 0,06$ & $0,42 \pm 0,05$ & $0,11 \pm 0,09$ \\
\hline FUA & - & 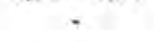 & $0,33 \pm 0,13$ & $0,98 \pm 0,00$ & $0,75 \pm 0,03$ & $0,38 \pm 0,10$ \\
\hline FVW & - & - & - & $0,39 \pm 0,11$ & $-0,33 \pm 0,09$ & $0,93 \pm 0,02$ \\
\hline FUA* & - & - & - & - & $0,70 \pm 0,03$ & $0,46 \pm 0,08$ \\
\hline $\mathrm{TZ}$ & - & - & - & - & $=$ & $-0,30 \pm 0,07$ \\
\hline
\end{tabular}

- Merkmal abgeleitet aus der erilsprechenden polymomialen Funktion fur die gesamte Testperiode

"Abkurzangen und Einheiten siehe Tabelle 1

Die genetischen Korrelationen von $\mathrm{r}_{\mathrm{g}}=0,96,0,98$ und 0,93 zu den entsprechenden gemessenen Merkmalen für TZ, FUA und FVW zeigen, dass diese Merkmale identisch sind. Die genetischen Korrelationen zwischen Tageszunahme, Futteraufnahme und Futterverwertung weisen ebenfalls kaum Unterschiede zwischen den gemessenen und den aus Funktionen abgeleiteten Werten auf. Die Futteraufnahme ist hoch mit der Tageszunahme korreliert $\left(\mathrm{r}_{\mathrm{B}}=0,68\right.$ bis 0,75$)$ und zeigt mittlere genetische Beziehun- 
gen mit der Rückenspeckdicke $\left(r_{\mathrm{g}}=0,51\right.$ und 0,47$)$ und der Futterverwertung $\left(r_{\mathrm{g}}=0,33\right.$ bis 0,46 ). Obwohl die Tageszunahme eine mittlere genetische Beziehung zur Rückenspeckdicke aufweist $\left(r_{\mathrm{B}}=0,39\right.$ und 0,42$)$, führt eine selektive Erhöhung der Zunahmeleistung zu einer verbesserten Futterverwertung $\left(r_{g}=-0,29\right.$ bis $\left.-0,39\right)$,

\section{Genetische Beziehumgen zwischen Merkmalen der gesamten Periode und in den Priafaloschanitten}

Die in Tabelie 7 zusammengefassten genetischen Korrelationen der durchschnittlichen Futteraufnahme in einzelnen Perioden zeigen in den Perioden zwei, drei und vier fast identische Schätzwerte zur Rückenspeckdicke $\left(\mathrm{r}_{\mathrm{B}}=0,40\right.$ bis 0,41$)$ und zur Futterverwertung $\left(r_{g}=0,46\right.$ bis 0,49). Dagegen steigen die genetischen Beziehungen der Futteraufnahme in diesen Perioden zu der Gesamttageszunahme $\left(r_{\mathrm{g}}=0,55\right.$ bis 0,66$)$ und der Gesamtfutteraufnahme $\left(r_{\mathrm{g}}=0,87\right.$ bis 0,97$)$ geringfügig an. Die genetischen Korrelationen der Futteraufnahme der ersten und letzten Prüfperiode sind deutlich niedriger und weisen insbesondere in der ersten Prüfperiode einen deutlich höheren Standardfehler auf.

Für die Tageszunahmen der einzelnen Perioden wurden die höchsten genetischen Korrelationen zu Rückenspeckdicke, Gesamttageszunahme und Gesamtfutteraufnahme in Periode zwei geschätzt $\left(\mathrm{r}_{\mathrm{g}}=0,42,0,91\right.$, und 0,66$)$. Während in den Perioden drei und vier die Korrelationen der jeweiligen Tageszunahme zur Gesamttageszunahme nur leicht absinken $\left(r_{\mathrm{g}}=0,86\right.$ und 0,80$)$ ist bei der Rückenspeckdicke $\left(r_{\mathrm{g}}=0,37\right.$ und 0,29$)$ und der Gesamtfutteraufnahme $\left(r_{\mathrm{g}}=0,50\right.$ und 0,40$)$ eine deutliche Verminderung in der genetischen Beziehung zur Tageszunahme im Zeitablauf zu verzeichnen. Die Tageszunahme in der letzten Periode ist unabhängig von der Rückenspeckdicke und weist nur eine niedrige Korrelation zur Gesamtfutteraufnahme auf. Die Beziehung der Tageszunahme in Periode fün zur Gesamttageszunahme ist, verglichen mit den Schätzwerten vorheriger Perioden, deutlich vermindert $\left(r_{\mathrm{g}}=0,47\right)$.

\section{Tabelle 7}

Genetische Korrelationen $\left(\mathrm{r}_{\mathrm{B}}\right)$ zwischen Mastleistungstnerkmalen fur den gesamten Prufzeitraum und Mastleistungsmerkmalen in den einzelnen Prufperioden (Genetic correlations $\left(r_{g}\right)$ of performance traits of entire test period with performance traits in each test period)

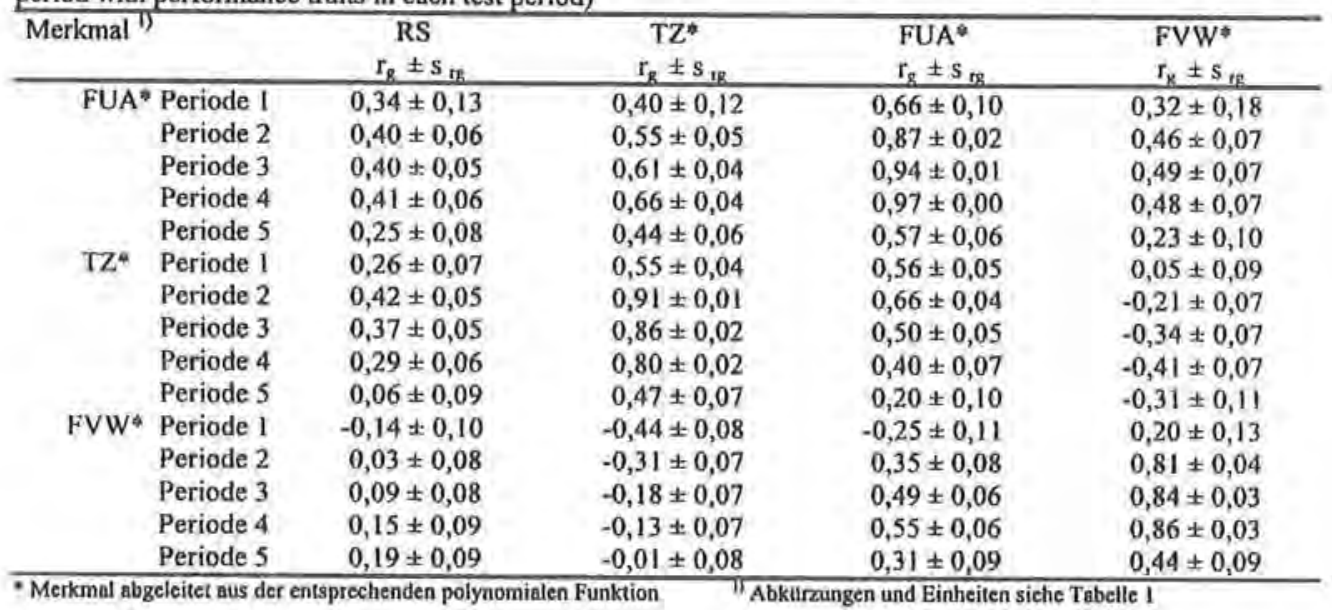


Die genetischen Beziehungen zwischen Tageszunahme und Gesamtfutterverwertung verbessert sich bis zur vierten Periode von 0,05 auf $-0,41$. In der ersten Periode ist die Abschnittszunahme in gleichem Maße mit der Gesamttageszunahme und der Gesamtfutteraufnahme korreliert $\left(\mathrm{r}_{\mathrm{g}}=0,55\right.$ und 0,56$)$ und niedrig zur Rückenspeckdicke $\left(\mathrm{r}_{\mathrm{g}}=\right.$ 0,26 ), während keine Beziehung zur Gesamtfutterverwertung besteht.

Die genetischen Korrelationen zwischen Futterverwertung in Abschnitten und der Gesamtfutteraufnahme zeigen eine Vorzeichenwechsel von der ersten zu den folgenden Perioden. Die genetischen Korrelationen zwischen Abschnittsfutterverwertung und der Gesamttageszunahme weisen in Periode eins die höchste züchterisch erwünschte $\mathrm{Bc}$ ziehung auf. In den folgenden Abschnitten nimmt die Beziehung ab und ist im letzten Abschnitt nicht vorhanden. Ein umgekehrte Entwicklung der genetischen Korrelationen konnte, wenn auch auf niedrigem Niveau, bei der Rückenspeckdicke beobachtet werden. Während in den ersten drei Perioden keine signifikanten genetischen Beziehungen bestehen, verschlechtert sich in den folgenden Perioden die Futterverwertung mit zunehmender Rückenspeckdicke.

\section{Genetische Bexiehungen zwischen Periodeninformationen}

Hohe genetische Korrelationen zwischen der beobachteten Futteraufnahme in einzelnen Testwochen und der geschätzten Futteraufnahme in den entsprechenden Zeiträumen wurden von SCHULZE et al. (2000) geschätzt. In jener, wie in der vorliegenden Auswertung kamen Polynome zur Anwendung, die keine Extrapolation erlauben. Da das Ziel der Untersuchung die Auswertung der genetischen Beziehungen in identischen Zeiträumen war, musste die Tageszunahme für die entsprechen Perioden definiert werden und führte zu einer Verschiebung der Zeiträume zwischen beobachteter und geschätzter Abschnittszunahme. Daher sind nur die genetischen Beziehungen zwischen den geschätzten Merkmalen in einzelnen Perioden dargestellt.

Die genetischen Beziehungen der Futteraufnahme zu Tageszunahme und Futterverwertung für einzelne Perioden sind in Tabelle $8 \mathrm{zu}$ sehen.

Tabelle 8

Genetische Korrelationen $\left(r_{b}\right)$ zwischen der Futteraufinahme und Tageszunahme sowie der Futterverwertung für die einzelnen Prufperioden (Genetic correlations $\left(r_{b}\right)$ of daily feed intake with daily gain as well as with food conversion ratio for each lest period)

\begin{tabular}{|c|c|c|c|c|c|c|}
\hline \multirow{2}{*}{\multicolumn{2}{|c|}{ Merkmal "I }} & \multicolumn{5}{|c|}{ FUA * } \\
\hline & & Periode 1 & Periode 2 & Periode 3 & Periode 4 & Periode 5 \\
\hline \multirow{11}{*}{$\mathrm{TZ}^{*}$} & & & & & $0.40+0.06$ & $=\frac{0.09}{48}$ \\
\hline & ae & & & & $067 \pm 0.03$ & $0,12 \pm 0,09$ \\
\hline & Periode 2 & $0,33 \pm 0,11$ & & $0,88 \pm 0,03$ & & $0,36 \pm 0,07$ \\
\hline & Periode 3 & $0,08 \pm 0,13$ & $0,43 \pm 0,05$ & $0,54 \pm 0,04$ & $0,61 \pm 0,04$ & $0,42 \pm 0,06$ \\
\hline & Periode 4 & $0,00 \pm 0,14$ & $0,28 \pm 0,06$ & $0,41 \pm 0,05$ & $0,53 \pm 0,05$ & $0,50 \pm 0,06$ \\
\hline & Periode 5 & $0,08 \pm 0,19$ & $0,01 \pm 0,09$ & $0,06 \pm 0,08$ & $0,21 \pm 0,09$ & $0,42 \pm 0,06$ \\
\hline & "Periode 1 & $-0,11 \pm 0,20$ & $-0,10 \pm 0,09$ & $-0,14 \pm 0,09$ & $-0,24 \pm 0,09$ & $-0,33 \pm 0,11$ \\
\hline & Periode 2 & $0,51 \pm 0,12$ & $0,55 \pm 0,06$ & $0,44 \pm 0,06$ & $0,21 \pm 0,08$ & $-0,28 \pm 0,09$ \\
\hline & Periode 3 & $0,51 \pm 0,12$ & $0,63 \pm 0,05$ & $0,56 \pm 0,05$ & $0,38 \pm 0,07$ & $-0,12 \pm 0,10$ \\
\hline & Periode 4 & $0,28 \pm 0,16$ & $0,53 \pm 0,06$ & $0,56 \pm 0,05$ & $0,52 \pm 0,06$ & $0,19 \pm 0,10$ \\
\hline & Periode 5 & $-0,33 \pm 0,17$ & $0,02 \pm 0,09$ & $0,21 \pm 0,08$ & $0,42 \pm 0,08$ & $0,56 \pm 0,08$ \\
\hline
\end{tabular}

Der Verlauf der genetischen Korrelationen zwischen Futteraufnahme und Tageszunahme deutet darauf hin, dass eine Selektion auf hohe Abschnittstageszunahme nicht 
nur mit einer hohen Futteraufnahme in der entsprechenden Periode verbunden ist, sondern auch in den nachfolgenden Perioden zu einer erhöhten korrelierten Futteraufnahme führt (Korrelationen oberhalb der Diagonale). Andererseits führt eine Selektion auf hohe Futteraufnahme zwar zu einer hohen Tageszunahme im entsprechenden Abschnitt, wird jedoch nicht im gleichen Ausmaß in einer hohen Tageszunahme in den folgenden Abschnitten resultieren (Korrelationen unterhalb der Diagonale). Die höchste genetische Korrelation zwischen Tageszunahme und Futteraufnahme in entsprechenden Perioden (auf der Diagonale) wurde in der zweiten Periode geschätzt $\left(r_{g}=\right.$ $0,62)$ und nimmt in den folgenden Perioden bis auf $r_{g}=0,42 \mathrm{ab}$. Die Futterverwertung in der ersten Periode weist nur eine niedrige Beziehung zur Futteraufnahme auf und ist ebenso wie alle Schätzwerte der Futteraufnahme der ersten Periode mit einem höheren Schätzfehler verbunden. Fast identische Korrelationen zwischen Futteraufnahme und Futterverwertung sind in den entsprechenden Abschnitten der zweiten bis fünften $\mathrm{Pe}$ riode $\mathrm{zu}$ verzeichnen $\left(\mathrm{r}_{\mathrm{g}}=0,52\right.$ bis 0,56$)$. Genetische Korrelationen in ähnlicher Grössenordnung wurden weiterhin zwischen der Futteraufnahme in den Perioden zwei und drei und der Futterverwertung in den Perioden zwei bis vier geschätzt.

In Tabelle 9 sind die genetischen Korrelationen zwischen Tageszunahmen und Futterverwertung in den einzelnen Perioden dargestellt.

Tabelle 9

Genetische Korrelationen $\left(\mathrm{r}_{\mathrm{g}}\right)$ zwischen Tageszunahmen und Futterverwertung in den einzelnen Prufperioden (Genetic correlations $\left(r_{k}\right)$ between daily gain and food conversion ratio for each test period)

\begin{tabular}{|c|c|c|c|c|c|}
\hline \multirow[t]{3}{*}{ Merkmal ") } & \multicolumn{5}{|c|}{$\mathrm{TZ}^{*}$} \\
\hline & Periode 1 & Periode 2 & Periode 3 & Periode 4 & Periode 5 \\
\hline & $r_{g} \pm s_{t g}$ & $r_{8} \pm s_{B 8}$ & $r_{8} \pm s_{08}$ & $r_{u} \pm s_{M g}$ & $\mathrm{r}_{\mathrm{g}} \pm \mathrm{s}_{\mathrm{rg}}$ \\
\hline FVW* Periode 1 & $-0,84 \pm 0,03$ & $-0,49 \pm 0,07$ & $-0,09 \pm 0,09$ & $0,08 \pm 0,10$ & $0,03 \pm 0,13$ \\
\hline Periode 2 & $-0,03 \pm 0,09$ & $-0,32 \pm 0,06$ & $-0,37 \pm 0,09$ & $-0,33 \pm 0,07$ & $-0,03 \pm 0,11$ \\
\hline Periode 3 & $0,39 \pm 0,07$ & $-0,10 \pm 0,07$ & $-0,38 \pm 0,06$ & $-0,45 \pm 0,06$ & $-0,17 \pm 0,10$ \\
\hline Periode 4 & $0,49 \pm 0,07$ & $0,07 \pm 0,08$ & $-0,26 \pm 0,07$ & $-0,44 \pm 0,07$ & $-0,38 \pm 0,10$ \\
\hline Periode 5 & $0,09 \pm 0,10$ & $0,28 \pm 0,08$ & $0,22 \pm 0,08$ & $0,01 \pm 0,09$ & $-0,51 \pm 0,09$ \\
\hline
\end{tabular}

Auffällig ist die hohe Korrelation zwischen beiden Merkmalen in der ersten Periode $\left(\mathrm{r}_{\mathrm{g}}\right.$ $=-0,84)$. Die genetischen Korrelationen auf der Diagonale in den folgenden Perioden sind deutlich niedriger $\left(r_{g}=-0,32\right.$ bis $\left.-0,51\right)$. Eine verbesserte Futterverwertung führt genetisch nicht nur zu einer hohen Zuwachsleistung in der entsprechenden Periode sondern auch zu einer höheren Tageszunahme in der nachfolgenden Periode, wie an den Korrelationskoeffizienten oberhalb der Diagonale zu erkennen ist. Die umgekehrte Schlussfolgerung, dass eine hohe Tageszunahme in der nachfolgenden Periode über genetische Beziehungen zu einer verbesserten Futterverwertung führt, ist jedoch nicht in dem Maße gegeben (Korrelationen unterhalb der Diagonale).

\section{Diskussion}

\section{Verlauf der Futteraufnahme und des Wachstums.}

Zur Beschreibung der individuellen Futteraufnahme und der individuellen Lebendmasseentwicklung als Funktion des Alters erwiesen sich Polynome zweiten und dritten Grades als geeignet. Ähnliche Darstellungen des Verlaufs der Futteraufnahme und Zunahme wie in Abbildung 1 finden sich bei (KRIETER 1986; SCHMIDT et al., 1991: 
ANDERSEN und PEDERSEN, 1996). In diesen Arbeiten wurden, basierend auf Daten aus gewichtsabhängigen Leistungsprüfungen, die Futteraufnahme, Tageszunahme oder die Lebendmasseentwicklung als Funktionen des Gewichtes oder Testtages mit Polynomen zweiten, dritten oder vierten Grades beschrieben. Eine lineare Entwicklung der Futterverwertung wurde auch von KRIETER (1986) zwischen Lebendgewicht und Futterverwertung im Gewichtsabschnitt zwischen 30 und $100 \mathrm{~kg}$ beobachtet.

Aus der Analyse des Wendepunktes der Wachstumskurve im Abschnitt 30-100 kg schlussfolgerten SCHMIDT et al. (1991), dass sowohl der Schlachtkörperwert als auch die Zuwachsleistung in einzelnen Abschnitten der Mast durch das Gewicht im Wendepunkt beeinflusst werden. Dies steht in guter Übereinstimmung mit den in den Abbildungen 2 und 3 aufgezeigten Kurvenverläufen. Unabhängig von Zunahmeniveau zeigen die Tiere mit vermehrter Speckauflage einen steileren Anstieg in den Zunahmen und erreichen ca. 14 Tage früher ein Maximum als die mageren Tiere beider Zunahmeniveaus (Abb. 3). Der Verlauf der Futteraufnahme unterscheidet sich ebenfalls zwischen mageren und fetten Tieren (Abb, 2). Allerdings ist eine reduzierte Futteraufnahme im letzten Abschnitt, die von SCHMIDT et al. (1991) als Ursache für einen hohen Muskelfleischanteil genannt wurde, nicht nur die Ursache für eine geringe Speckauflage $(\mathrm{TZ}<=975 \mathrm{~g}$ und $\mathrm{RS}<10,1 \mathrm{~mm}$ ), sondern die Tiere mit hohem Ruckenspeck und hohem Wachstum weisen einen steileren Anstieg zu Beginn der Mast und auch eine deutlich stärkere Krümmung der Futteraufnahmekurve auf. Nach KRIETER und KALM (1989) erreichen Schweine mit einer hohen Wachstumsrate im frühen Mastabschnitt ihre maximale Wachstumsrate eher und bei einem niedrigeren Lebendgewicht, sind frühreifer und haben als adulte Tiere ein niedrigeres Lebendgewicht. Uberträgt man diese Aussage auf die vorliegenden Tiergruppen, so würden die Tiere mit erhöhtem Rückenspeck als frühreif eingestuft, während das Wachstumspotential der mageren Tiere nicht ausgeschöpft wird, da die maximale Wachtumsrate erst am Ende der Pribfperiode erreicht wird.

\section{Heritabilitäten für gesamten Zeitraum}

Hohe Heritabilitäten wurden für die Merkmale Rückenspeckdicke $\left(h^{2}=0,50\right)$ und die aus den Funktionen abgeleiteten Merkmale Futteraufnahme, Tageszunahme und Futterverwertung $\left(\mathrm{h}^{2}=0,40,0,55\right.$ und 0,39$)$ für die gesamte Periode geschätzt. Diese Erblichkeiten befinden sich bis auf die Tageszunahme im Bereich der Schätzwerte von $h^{2}$ $=0,38$ bis 0,65 für die Rückenspeckdicke, $h^{2}=0,16$ bis 0,42 für die Futteraufnahme, $h^{2}$ $=0,25$ bis 0,49 für die Tageszunahme sowie $h^{2}=0,12$ bis 0,51 für die Futterverwertung in den Untersuchungen von DE HAER (1992), VON FELDE et al. (1996), LABROUE et al. (1997), HALL et al. (1999) sowie EISSEN (2000). In diesen Arbeiten wurde die Futteraufnahme durch elektronische Futterstationen erhoben.

Die Angaben dieser Autoren zu den genetischen Korrelationen zeigten die geringste Schwankung zwischen der Futteraufnahme und der Tageszunahme $\left(r_{g}=0,61\right.$ bis 0,88$)$, während sowohl die Korrelationen zwischen Tageszunahme und Rückenspeckdicke $\left(\mathrm{r}_{\mathrm{g}}\right.$ $=0,23$ bis 0,53$)$, als auch zwischen Futteraufnahme und Rückenspeckdicke $\left(r_{g}=0,35\right.$ bis 0,78$)$ größere Unterschiede aufweisen. Die größte Spannweite konnte bei den Korrelationen der Futterverwertung mit der Tageszunahme $\left(r_{\mathrm{g}}=0,13\right.$ bis $\left.-0,64\right)$, mit der Rückenspeckdicke $\left(r_{\mathrm{g}}=-0,22\right.$ bis 0,51$)$ und mit der Futteraufnahme $\left(r_{\mathrm{g}}=-0,06\right.$ und 
$0,65)$ beobachtet werden. Die genetischen Korrelationen zwischen den Mastleistungsmerkmalen in der vorliegenden Untersuchung liegen im Bereich dieser Literaturangaben und zeigen an, dass eine erhöhte Futteraufnahme zwar zu einer besseren Tageszunahme $\left(r_{g}=0,74\right)$ aber auch zu einer höheren Rülckenspeckdicke $\left(r_{g}=0,51\right)$ fuhrt. Eine züchterische Verbesserung der Futterverwertung ist durch Selektion auf erhöhte $\mathrm{Zu}$ wachsleistung $\left(r_{g}=-0,39\right)$ zu erzielen, während eine Steigerung der Futteraufnahme $\left(r_{g}\right.$ $=0,46) \mathrm{zu}$ einem unerwünschten Anstieg der Futterverwertung fuhrt. Sowohl die Tageszunahme als auch die Futteraufnahme sind in züchterisch unerwünschter Weise mit der Rückenspeckdicke genetisch korreliert, so dass mit diesen Merkmalen über den gesamten Zeitraum aufgrund der genetischen Beziehungen eine Erhöhung der Futteraufnahme bei gleichzeitiger Optimierung des Schlachtkörperwertes und der Zuwachsleistung schwer zu realisieren ist.

\section{Genetische Parameter zwischen einzelnen Abschnitten}

Eine mit der Testdauer steigende Erblichkeit der Futteraufnahme mit maximalen $\mathrm{He}$ ritabilitäten im mittleren Testabschnitt, wie in der vorliegenden Untersuchung, konnte auch in den Arbeiten von RÖHE et al. (1994), VON FELDE et al. (1996) und SCHULZE et al. (2000) gefunden werden. Dagegen schätzten HALL et al. (1999) die höchste Erblichkeit fur Futteraufnahme zu Beginn der Mastperiode und fanden in Übereinstimmung mit SCHMIDT et al. (1991) die niedrigste Erblichkeit fur Futteraufnahme im letzten Auswertungsabschnitt. SCHNYDER et al. (1999) fanden bei einer Auswertung von zwei Rassen einen kontinuierlichen Anstieg in den Erblichkeiten von $h^{2}=0,09$ und 0,13 in der ersten Testwoche bis $h^{2}=0,23$ und 0,32 in der letzten Testwoche, Die in der vorliegenden Analyse geschätzte niedrige Heritabilität in Periode 1 $\left(h^{2}=0,18\right)$ im Vergleich zu den Schätzwerten in den folgenden Perioden $\left(h^{2}=0,39\right.$ bis $0,46)$ wurde auch von SCHULZE et al. $(2000)$ beobachtet. Diese Autoren erklärten dies mit einer nicht ausreichend langen Eingewöhnungsphase an elektronischen Futterstationen. HALL et al. (1999) konnten, ohne Berücksichtigung der ersten Testwoche an den elektronischen Futterstationen, keine Differenzen in den Heritabilitäten zwischen der ersten und nachfolgenden Perioden finden.

Über die Erblichkeit der Tageszunahmen in den einzelnen Mastabschnitten oder Prüfperioden liegen wenig Untersuchungen vor. SCHMIDT et al. (1991) schätzten die Erblichkeit der Tageszunahme in drei Mastabschnitten $(30-53 \mathrm{~kg}, 54-77 \mathrm{~kg}$ und 78 $100 \mathrm{~kg}$ ) auf der Basis eines Vatermodells. Diese Autoren konnten hohe Erblichkeiten für die ersten beiden Abschnitte finden $\left(h^{2}=0,37\right.$ und 0,43$)$, während im dritten $A b$ schnitt ein leichter Abfall festzustellen war $\left(h^{2}=0,26\right)$. Ein ähnlicher Verlauf der Heritabilitäten für Tageszunahme in Perioden von 12 Tagen konnte in der vorliegenden Untersuchung beobachtet werden. Ein leichter Anstieg der Erblichkeit von der ersten $\left(h^{2}=0,50\right)$ zur folgenden Periode $\left(h^{2}=0,56\right)$ und ein deutlicher Abfall im letzten Abschnitt $\left(h^{2}=0,37\right)$.

Die Heritabilitäten der Futterverwertung in Tabelle 5 waren in den mittleren drei Perioden hoch, während in den Perioden eins und fünf niedrigere Erblichkeiten geschätzt wurden. Dies kann durch die erhöhten genetischen und umweltbedingten Variationen in diesen Perioden verursacht sein und kann in dem sehr unterschiedlichen Verlauf der Futterverwertung (Abb. 4) in den Perioden eins und funf begrlindet sein. SCHMIDT et 
al. (1991) fanden Erblichkeiten in drei Gewichtsabschnitten von $\mathrm{h}^{2}=0,36,0,36$ und 0,26 mit dem niedrigsten Schätzwert im letzten Abschnitt.

\section{Genetische Korrelationen der Mastleistungsmerkmale in Abschnitten}

Die hohen genetischen Korrelationen zwischen Abschnittsinformationen und den Mastleistungsmerkmalen des gesamten Zeitraums zeigen, dass die Perioden zwei, drei und vier keinen hohen Informationsgewinn gegenüber der Verwendung des entsprechenden Gesamtmerkmals bringen. Dagegen sind die genetischen Korrelationen in den Perioden eins und füf deutlich niedriger. Die Korrelationen zwischen den Merkmalen in den Tabellen 8 und 9 verdeutlichen weiterhin, dass insbesondere eine höhere Futteraufnahme in Periode eins mit einer besseren Tageszunahme in genetischer Bezichung steht $\left(r_{B}=0,56\right)$ und diese erhöhte Tageszunahme zu einer maßgeblichen Verbesserung der Futterverwertung fuhrt $\left(r_{g}=-0,84\right)$, da die genetische Korrelation zwischen der Tageszunahme in dieser ersten Periode zur Rückenspeckdicke niedrig ist $\left(\mathrm{r}_{\mathrm{g}}=0,26\right)$. In den nachfolgenden Perioden sind die Korrelationen der Futteraufnahme mit der Futterverwertung in den entsprechenden Perioden relativ konstant $\left(r_{B}=0,52\right.$ bis 0,56$)$ und leicht fallend zwischen Futteraufnahme und Tageszunahme $\left(r_{g}=0,62\right.$ bis 0,42$)$. Der stetige Anstieg der Korrelationen zwischen Tageszunahme und Futterverwertung von Periode zwei zu Periode fünf $\left(r_{g}=-0,32\right.$ bis $\left.-0,51\right)$ zeigt ein erwünschtes spätes Maximum im Wachstum wie in Abbildung 3 an.

SCHMIDT (1988) konnte ebenfalls die höchsten genetischen Beziehungen zwischen Tageszunahme und Futterverwertung im ersten Mastabschnitt feststellen $\left(r_{g}=-0,64\right)$ und ein Absinken zum letzten Abschnitt $\left(r_{g}=-0,57\right.$ und $\left.-0,51\right)$ hin verzeichnen. Allerdings waren die Differenzen der Korrelationen zwischen den Mastabschnitten gering. Zwischen der Futteraufnahme in einzelnen Abschnitten und der entsprechenden Futrerverwertung bestand keine genetische Beziehung, während die genetischen Korrelationen zwischen Futteraufnahme und Tageszunahmen von 0,67 im ersten Mastabschnitt auf 0,93 im dritten Mastabschnitt anstieg, KRIETER (1986) schätzte identische Korrelationen zwischen Futteraufnahme und Zunahme in entsprechenden Gewichtsabschnitten $\left(r_{\mathrm{g}}=0,78,0,84\right.$ und 0,83$)$. Während die genetische Beziehung zwischen Tageszunahme und Futterverwertung mit steigendem Gewicht abnahm $\left(r_{g}=-0,66\right.$ bis 0,27 ), konnte ein Ansteigen der genetischen Korrelationen zwischen Futteraufnahme und Futterverwertung im letzten Gewichtsabschnitt $\left(r_{g}=-0,05,0,04,0,30\right)$ festgestellt werden. Sowohl in der Arbeit von SCHMIDT (1988) als auch in der Arbeit von KRIETER (1986) wurde die individuelle Futteraufnahme nicht direkt erfasst, sondern aus der Gruppenfutteraufnahme und der individuellen Zuwachsleistung abgeleitet. Dies kann eine Ursache für die, verglichen mit den Ergebnissen der vorliegenden Untersuchung, niedrigeren Korrelationen zwischen Futteraufnahme und Futterverwertung sowie den höheren Korrelationen zwischen Tageszunahme und Futteraufnahme in einzelnen Abschnitten gewesen sein.

Schlussfolgerungen

Die Ergebnisse dieser Untersuchung zeigen, dass es deutliche Unterschiede im Verlauf der individuellen Futteraufnahme- und Wachstumskurven gibt, die in einzelnen Ab- 
schnitten der Leistungsprüfung zu Unterschieden in der Futterverwertung führen. Eine hohe Futteraufnahme in der ersten Periode der Leistungsprifung erweist sich als notwendig, um eine hohe Zuwachsleistung zu erreichen. In den nachfolgenden Perioden zeigt eine maximale Futteraufnahme nachteilige Auswirkungen in Bezug auf ein hohes Rückenspeckwachstum. Die Tiere mit einem erhöhten Speckmaß erreichen die maximale Zuwachsrate früher als Tiere mit einer niedrigeren Rückenspeckdicke.

Zudem wird ein frühes Erreichen der maximalen Zuwachsrate mit einem niedrigeren Endgewicht und einer früher eintretenden Geschlechtsreife in Verbindung gebracht (KRIETER und KALM, 1989). Daher ist ein lang andauernder Anstieg der täglichen Zuwachsrate anzustreben, um die von der zweiten bis zur fünften Periode ansteigende günstige genetische Beziehung zur Futterverwertung auszunutzen. Jedoch muss in Mutterlinien beachtet werden, dass keine negativen Auswirkungen auf die eintretende Geschlechtsreife und die nachfolgende Fruchtbarkeitsleistung auftreten. So konnten KARSTEN et al. (2000) züchterisch unerwünschte geringe Korrelationen zwischen der Futterverwertung eigenleistungsgeprüfter Eber und der Fruchtbarkeitsleistung der weiblichen Nachkommen im ersten Wurf nachweisen. Auch die Futteraufnahme sollte ausreichend sein, da eine signifikante Beziehung zur Fruchtbarkeit festgestellt wurde.

Zusammenfassend zeigen die genetischen Beziehungen zu dem Effizienzparameter Futterverwertung an, dass eine hohe Futteraufnahme am Anfang der Mast mit zunehmender Limitierung mit fortdauernder Mast anzustreben ist. Zudem sollte das maximale Wachstum gegen Ende der Mast sein, um ein erhöhtes Fettwachstum zu vermeiden und die günstig werdenden genetischen Beziehungen zur Futterverwertung auszunutzen. Um das Wachstum und die Futteraufnahme während der Mast zu optimieren, ist daher die Verwendung von Abschnittsinformationen notwendig.

Durch eine unterschiedliche Bewertung der einzelnen Abschnitte kann die Zielsetzung einer Erhöhung der Effizienz der Mastleistung und ein Anstieg der Futteraufnahme am Anfang der Mast erzielt werden.

\section{Literatur}

AGRICULTURAL RESEARCH COUNCIL (ARC):

The nutrient requirements of pigs. Commonwealth Agricultural Bureaux, Slough, (1981)

ANDERSEN, S.; PEDERSEN, B.: Growth and food intake curves for group-housed gilts and castrated male pigs. Anim. Sci. 63 (1996),
$457-464$

DE HAER, L.C.M.:

Relevance of eating pattern for selection of growing pigs. PhD Thesis, Univ. Wageningen, The EISSEN, J,: Netherlands, 1992

Breeding for feed intake capacity in pigs. PhD Thesis, Univ, Wageningen, The Netherlands, 2000

HALL, A.D; HILL, W.G.; BAMPTON, P.R.; WEBB, A.J; Genetic and phenotypic parameter estimates for feeding pattern and performance traits in pigs. Anim. Sci., 68 (1999), 43-48

KARSTEN, S.; RÖHE, R.; SCHULZE, V.; LOOFT, H.; KALM, E.: Genetische Beziehungen zwischen individueller Futteraufnahme wathrend der Eigenleistungsprufung und Fruchtbarkeitsmerkmalen beim Schwein. Arch. Tierz, Dummerstorf 43 (2000), 451-461

KNAP, P.W.; VAN DER STEEN, H.A.M.:

Genetic aspects of growth curve shape in pigs. Proc. $5^{\text {th }}$ World Congr. Genet. Appl, Lives. Prod. 19 (1994), 193-196 


\section{KRIETER, J.:}

Entwicklung von Selektionsmethoden für das Wachstums- und Futteraufnahmevermögen beim Schwein. Diss., Univ. Kiel, 1986

KRIETER, J; KALM, E.:

Growth, feed intake and mature size in Large White and Pietrain pigs. J. Anim. Breed. Genet. 106 (1989), 300-311

LABROUE, F.; GUEBLEZ, R.; SELLIER, P.:

Genetic parameters of feeding behaviour and performance traits in group-housed Large White and French Landrace growing pigs. Genet. Sel. Evol. 29 (1997), 451-468

MISZTAL, 1: MTDFS, users notes, University of Illinois, USA (1993)

ROBÉRTSON, A.:

The sampling variance of the genetic correlation coefficient. Biometrics 15 (1959), 469-485

RÖHE, R.; VON FELDE, A,; LOOFT, H.; KRAPOTH, J; KALM, E.:

Effect of stage of growth on genetic parameters of feed intake in swine. Proc. $5^{\text {th }}$ World Congr. Genet, Appl. Lives. Prod. 19 (1994), 185-188

SCHMIDT, E.:

Analyse des Wachstums und seine Beziehungen zum Schlachtkörperwert beim Schwein. Diss., Univ. Kiel, 1988

SCHMIDT, E.; KRIETER, J.; KALM, E;

Wachstum beim Schwein: Vergleich von Funktionen sowie Verlauf von Tageszunahme und Futteraufnahme und deren Beziehung zum Schlachtkörperwert. Zichtungskunde 63 (1991), 306-316

SCHNYDER, U.; HOFER, A.; LABROUE, F; KÜNZI, N:

Genetic parameters of a random regression model for daily feed intake of performance tested French Landrace and Large White growing pigs. $50^{\text {th }}$ Annual Meeting of the EAAP, Zirich, Session G2.7 (1999)

SCHULZE, V., RÖHE, R., LORENZO BERMEJO, J., LOOFT, H., KALM, E.:

Genetic analysis of parameters of feed intake curves of performance tested boars. $5 \mathrm{I}^{\text {th }}$ Annual Meeting of the EAAP, Den Haag, Session G5.4 (2000)

VON FELDE, A; ROHE, R; LOOFT, H.; KALM, E.:

Genetic association between feed intake and feed intake behaviour at different stages of growth of group-housed boars. Livest. Prod. Sci. 47 (1996), 11-22

Eingegangen: 27.12.2000

Akzeptiert: 22.01 .2001

Anschriften der Verfasser

VOLKER SCHULZE, PD Dr. RAINER RÖHE, Prof. Dr. Dr. h.c. mult. ERNST KALM

Christian-Albrechts-Universităt zu Kiel

Institut fur Tierzucht und Tierhaltung

Olshausenstr. 40

D-24098 Kiel

Dr. HOLGER LOOFT

PIC Deutschland GmbH

Ratsteich 31

D-24837 Schleswig 